\title{
Isolated scapular tuberculosis: an uncommon presentation of skeletal tuberculosis
}

\author{
Ghanshyam Dev ${ }^{1}$, Shwait Sharma ${ }^{1}$, Mohd Ilyas ${ }^{2}$ \\ ${ }^{1}$ Department of Radiodiagnosis, Government. Medical College, Jammu, India \\ ${ }^{2}$ Department of Radiodiagnosis, Sher-i-Kashmir Institute of Medical Sciences, Srinagar, India
}

DOI: $10.18621 /$ eurj.387051

\begin{abstract}
Objective: The main objective of the study was to evaluate the radiological manifestations of isolated scapular tuberculosis.

Methods: A prospective study was performed wherein 50 patients with the complaint of swelling in the scapular region were evaluated using various imaging modalities for scapular tuberculosis. Patients having features of scapular tuberculosis were subjected to fine needle aspiration cytology (FNAC) and histopathology for confirmation of diagnosis.

Results: Four patients were diagnosed as having isolated scapular tuberculosis. All the four patients presented with lytic lesions in scapula with associated collections in adjacent muscles. All were subjected to antituberculosis therapy (ATT). In two patients drainage of abscess was done. All patients showed excellent response to treatment at 6 months follow-up.

Conclusion: Radiological investigations play a key role in early diagnosis of isolated scapular tuberculosis with excellent outcome with ATT and drainage of collection.
\end{abstract}

Keywords: Tuberculosis, lytic lesion, scapula, anti-tuberculosis therapy, musculoskeletal infections

Received: January 31, 2018; Accepted: February 23, 2018; Published Online: March 20, 2018

S capula is a broad flat, triangular bone lying obliquely over the posterior aspect of chest wall at the first seven ribs. Like any other bone scapula is also affected by congenital, traumatic, neoplastic and infectious process [1]. Infectious process of the scapula is represented by osteomyelitis of the scapula and is a rare diagnosis. Most of the cases of lytic lesion of the scapula with associated swelling are due to benign or malignant pathology affecting the scapula. Only few reports of tubercular involvement of the scapula are available. Tuberculosis continues to be a major cause of morbidity and mortality from infectious diseases worldwide more so in endemic areas.
Musculoskeletal infection may account for $10-30 \%$ of cases of extrapulmonary tuberculosis and overall for almost $2 \%$ of all cases of tuberculosis [2]. The spine is involved in approximately half of patients with musculoskeletal infections. The next most common is tuberculous arthritis followed by extra-spinal tuberculous osteomyelitis. Occurrence of tuberculosis in flat bones without any other foci is very rare. Less than $1 \%$ of skeletal tuberculosis occurs in the shoulder. Isolated scapular involvement in tuberculosis is very rare [3]. The aim of this study was to evaluate the radiological manifestations of isolated scapular tuberculosis. 


\section{METHODS}

This prospective study was performed over a period of two years (August 2015 to July 2017). Fifty patients with lytic lesion of the scapula along with associated swelling and pain in the shoulder and scapular region were evaluated with various radiological modalities including computed tomograhy (CT) scan, magnetic resonance imaging (MRI), and ultrasonography. After careful clinical history, physical examination and laboratory investigations, the patients were subjected to various radiological modalities for diagnosis of lytic lesion of the scapula. Out of the 50 patients, four patients were diagnosed with isolated tuberculosis of the scapula. The diagnosis was confirmed on histopathology. In all the patients a detailed clinical history, complete local and systemic examination was done followed by laboratory investigations. Informed consent was obtained from all the patients included in the study. Laboratory investigations included hemogram, total and differential counts, sputum exam, erythrocyte sedimentation rate (ESR) and Mantoux test. Radiological investigations included CT scan, MRI and ultrasonography. CT scan included contrast scan where required. MRI sequences included T1, T2, STIR and post-contrast sequences. All the patients were subjected to FNAC for confirmation of diagnosis on ZN staining \& histopathology. After confirmation of diagnosis, all the four patients were given antituberculosis therapy (ATT). In two out of four drainage of abscess was done for treatment. All the four patients showed excellent response to treatment at 6 months follow-up.

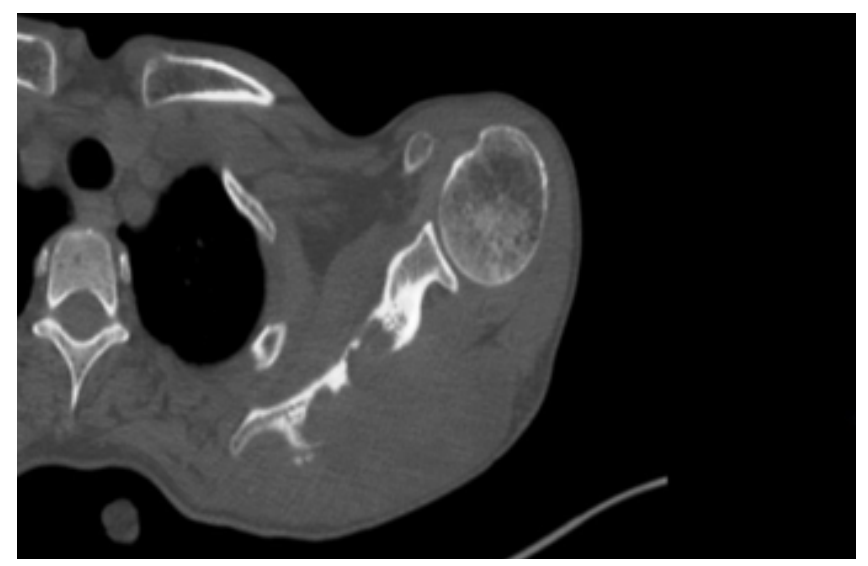

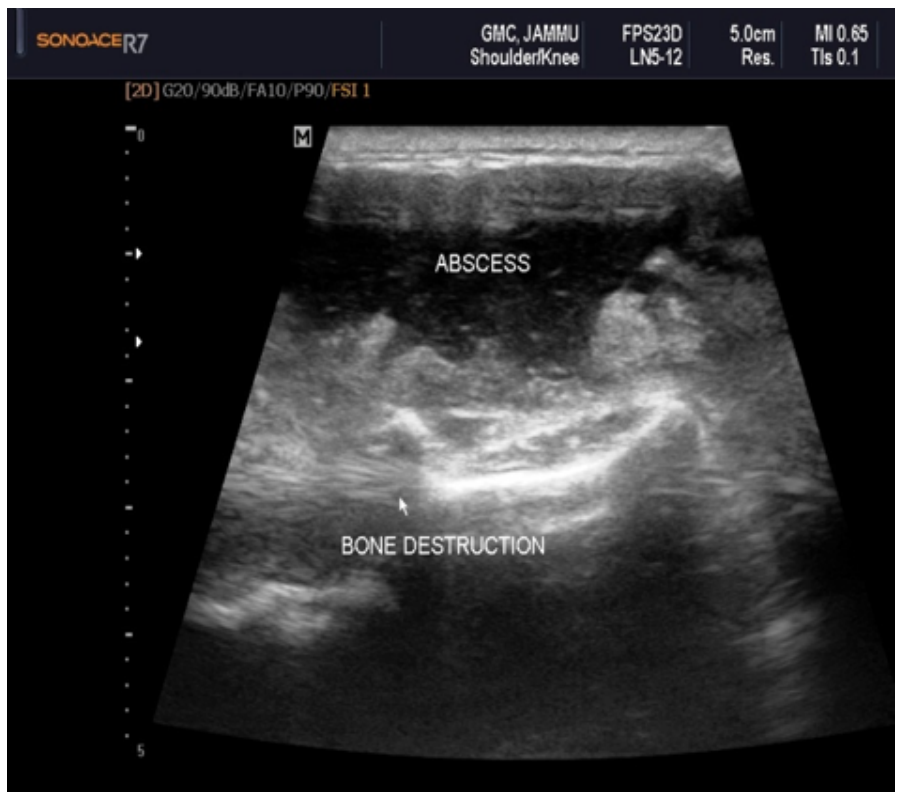

Figure 1. Linear ultrasound image of a patient with scapular tuberculosis showing scapular bone destruction with adjacent abscess in muscle.

\section{RESULTS}

All the four patients diagnosed with isolated scapular tuberculosis presented with swelling in the scapular region gradually increasing in size. In two patients it was associated with deep dull aching pain while in other two it was painless. One patient reported history of fever on \& off. No history of weight loss, fatigue, loss of appetite or cough was present in all the four patients. One out of four patients was diabetic. On physical examination, all patients presented with a lobulated swelling over left scapular region. The swelling was non tender with firm

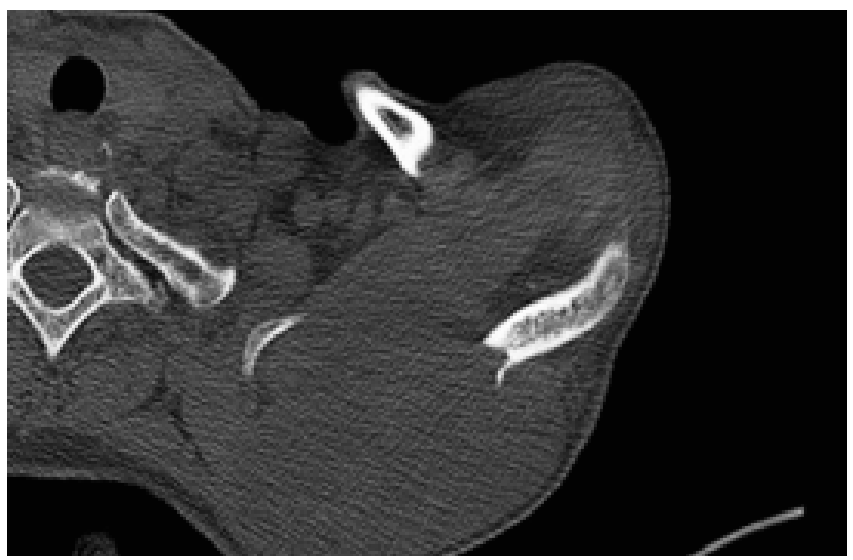

Figure 2. Non-contrast CT scan in axial plane in two patients showing lytic lesions with associated soft tissue collections. 


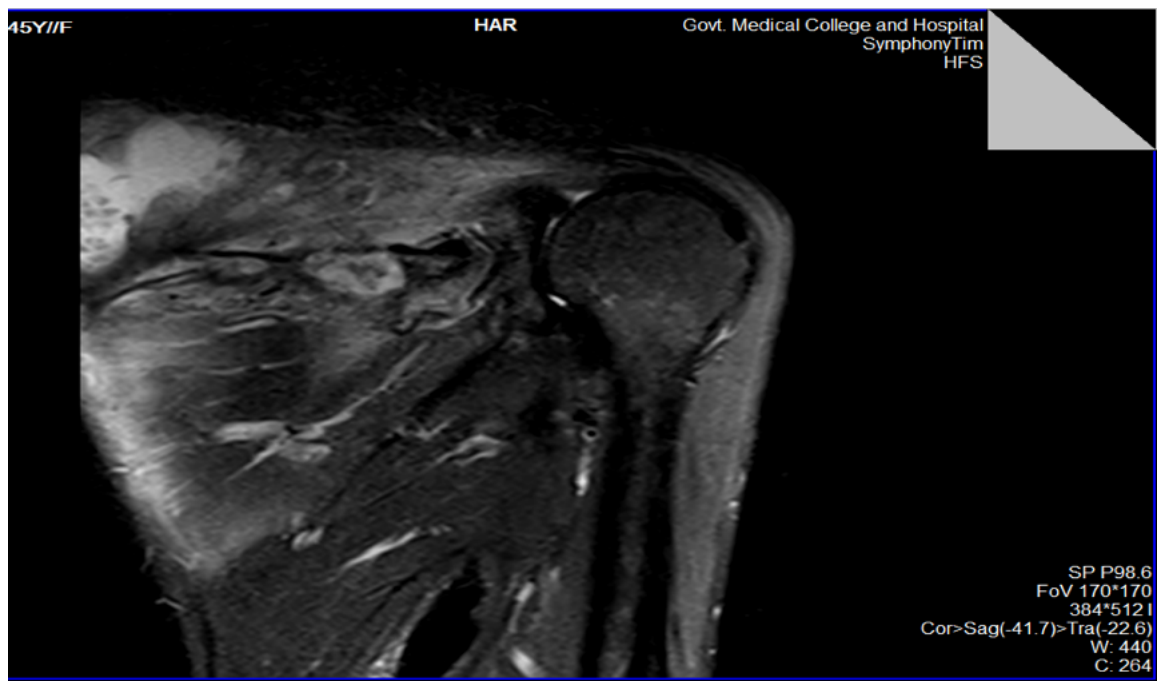

Figure 3. STIR weighted oblique coronal image of scapula showing scapular spine lytic lesion with associated edema in supraspinatous and infra-spinatous muscle and adjacent collection.

consistency. Laboratory investigations revealed raised ESR and positive qualitative C-reactive protein (CRP) in all the four patients.

$\mathrm{X}$ ray of the shoulder region revealed lytic lesion involving the scapula with associated soft tissue swelling. Scapular blade was involved in two patients, one showed involvement of inferior angle while in fourth one spine and blade was involved. Glenoid was not involved in any case. Shoulder joint was normal in all the four cases. No lesion was evident in humerus in all cases.

Ultrasonography of the swelling in all four patients revealed an echoic collection in soft tissues around the scapula with no internal vascularity on colour Doppler. Associated bony destruction was evident (Figure 1).

CT scan was done in two out of the four patients. Both patients revealed lytic lesion involving the scapula (Figure 2). One patient showed involvement of scapular blade while in other both scapular spine and

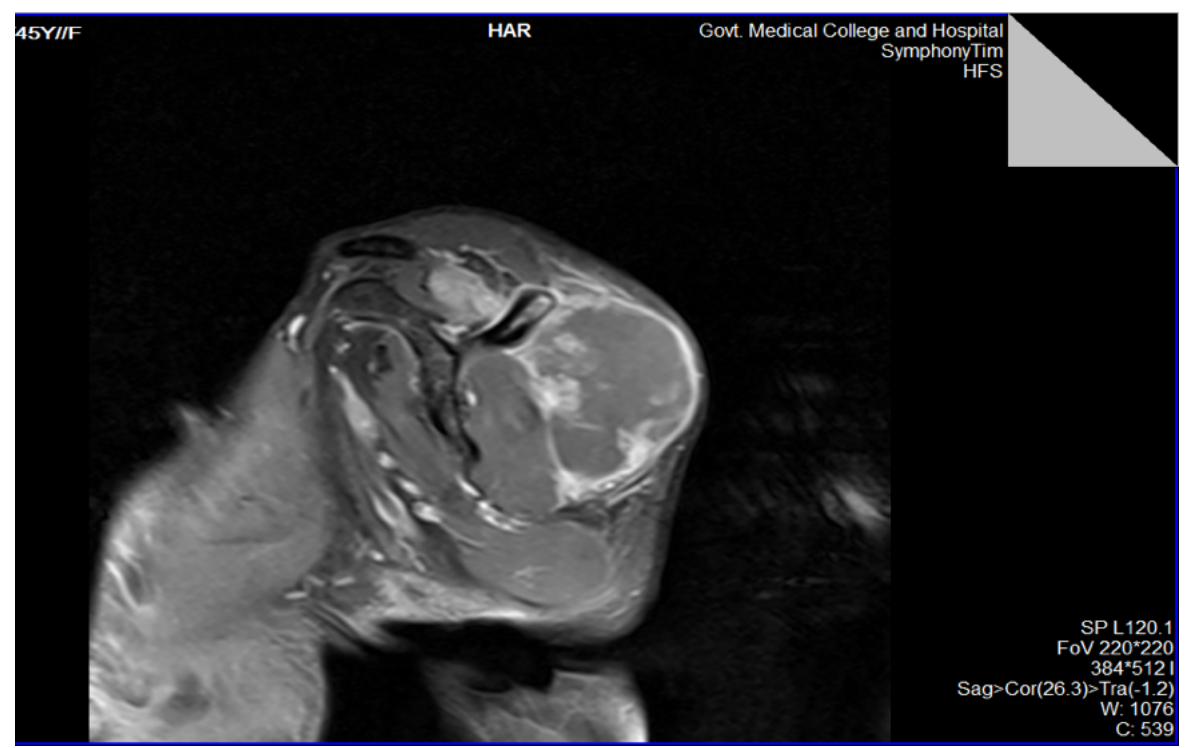

Figure 4. Post-contrast T1FS sagittal oblique MRI scan of the patients showing destruction of scapular blade and spine with associated collections in soft tissue. 
blade were involved. In both the patients, glenoid was not involved. Shoulder joint was normal. Upper end of the humerus was normal. Associated collections in adjacent soft tissue were noted in both patients.

Contrast enhanced MRI was done in one out of four patients. T1, T2, STIR and post-contrast sequences were taken in axial, sagittal and coronal planes (Figures 3 and 4). MRI revealed destruction of the scapular spine with associated collections in supraspinous fossa and in the axilla. Adjacent vertebral spine was normal.

The scapular tuberculosis is one of the rarest pathologies affecting the scapula and in the present study it accounted for only $8 \%$ patients. Three of these patients were males and one was female. The diabetic patient was female. The patients aged 34, 36, 50, and 48 years. None of these patients had any history of any pulmonary or extra-pulmonary tuberculosis. Three of these patients were living in the rural areas and one was from urban residence. All the investigations were effective in evaluation of the lesion.

\section{DISCUSSION}

Tuberculosis is an infection involving virtually every organ including skeletal system and is known since ages. Typical features of spinal tuberculosis have been seen in Egyptian mummies dating back to almost 4000 BC [4]. Musculoskeletal infection constitutes about $10-35 \%$ cases of extra-pulmonary tuberculosis and about $2 \%$ of all cases of tuberculosis. In the majority of patients, the spine is involved constituting about $50 \%$ cases. The next most common is tuberculous arthritis followed by extra-spinal tuberculous osteomyelitis [5]. Skeletal tuberculosis, especially isolated scapular tuberculosis, simulates a malignant lesion clinically and high suspicion is key to diagnosis [6]. Solitary tuberculous osteomyelitis involving flat membranous bones is a rare described entity. Very few cases of isolated scapular tuberculosis has been reported till date [7].

Clinically, scapular tuberculosis presents with pain and swelling in the scapular region. Restriction of movements of the shoulder may be present. Discharging sinus and constitutional symptoms are uncommon, making a diagnostic dilemma. Skeletal tuberculosis can appear as joint space narrowing, sub- chondral erosions, lytic lesions or articular osteopenia on plain radiographs [8]. Laboratory investigations reveal elevated ESR and CRP with lymphocytosis in few cases [2].

In our case series all the cases of scapular tuberculosis presented with pain and swelling in the scapular region. X-ray of the scapular region revealed lytic lesion of the scapula. Ultrasonography showed collection around the scapula. CT revealed lytic lesion of the scapula with adjacent collection in deep muscles around the scapula. MRI study revealed lysis of the scapula with adjacent peripherally enhancing collection.

Multimodality imaging approach play a key role in diagnosis of the scapular tuberculosis. High index of suspicion in appropriate clinical setting leads to early diagnosis in many cases enabling the timely initiation of therapy for better outcome. Surgical treatment of scapular tuberculosis involves drainage of collection. Sequestra of tuberculosis resolve with adequate ATT and surgical removal is not warranted [9]. Surgical removal is required in patients with giant sequestra or where the response to conservative treatment of 4 to 6 weeks is not satisfactory [10]. In our three out of four cases drainage of collection was done. However, surgical removal of sequestra was not required as all the four cases showed excellent response to drainage of collection and ATT. Differential diagnosis include other chronic skeletal infections such as brucellosis, actinomycosis, etc., and malignant pathologies including metastasis.

Tubercular osteomyelitis of the scapula is an extremely rare manifestation and atypical presentation at unusual site like the scapula, many a times may lead to misdiagnosis or delay in diagnosis [11]. The indolent nature of the disease and lack of constitutional symptoms often causes late presentation. Raised ESR and positive Monteux test are though consistent findings; these are not diagnostic of tuberculosis in endemic areas. Radiographic findings in tubercular osteomylitis include radiolucent lesion with irregular margin and surrounding sclerosis [12]. The cystic cavitary lesions on radiograph are highly nonspecific and simulate with pyogenic osteomylitis, fungal infection, metastasis, telengiectactic osteosarcoma, aneurysmal cyst, sarcoidosis, eosinophilic granuloma or chordoma. Differentiation of tuberculosis from all these 
differentials may not be possible without tissue biopsy. MRI scan may be sometime deceptive [2]. In the present study, the MR showed peripheral enhancement of the collection in the scapula which was higly reflective of an infective pathology but final diagnosis was formulated only on histopathological analysis.

\section{CONCLUSION}

To conclude, isolated scapular tuberculosis is a rare entity. Very few cases are reported earlier. Radiological investigations play a key role in early diagnosis of isolated scapular tuberculosis with excellent outcome with ATT and drainage of collection. High index of suspicion in cases of destructive scapular mass with associated collections in adjacent muscles in appropriate clinical setting can lead to early diagnosis supported by appropriate radiological investigations and confirmed on biopsy and histopathology.

\section{Conflict of interest}

The authors disclosed no conflict of interest during the preparation or publication of this manuscript.

\section{REFERENCES}

[1] Alvarez de Sierra Garcia B, Exposito Jimenez D, Bueno Horcajadas A, Olmedilla P, Sanz-Merino A, Martel Villagran J. Radiologic assessment of scapular region: US, MDCT and MR features. ECR 2012: C-1080, Madrid, Spain.

[2] Singh A, Chatterjee P, Pai MC, Chacko RT. Tuberculous osteomyelitis of the scapula masquerading as metastasis. J Radiol Case 2009;3:27-31.

[3] Mohan V, Danielsson L, Hosni G, Gupta RP. A case of tuberculosis of the scapula. Acta Orthop Scand 1991;62:79-80.

[4] Arriaza BT, Salo W, Aufderheide AC, Holcomb TA. Pre-Columbian tuberculosis in northern Chile: molecular and skeletal evidence. Am J Phys Anthropol 1995;98:37-45.

[5] Tuli SM. Tuberculosis of the Skeletal System (bones, joints, spine and bursal sheaths). 1st edn. Jaypee Brothers Medical Publishers (P) Ltd: New Delhi (India), 1993., p.121.

[6] Tsay MH, Chen MC, Jaung GY, Pang KK, Chen BF. Atypical skeletal tuberculosis mimicking tumor metastasis: report of a case. J Formos Med Assoc 1995; 94:428-31.

[7] Balaji G, Arockiaraj J, Roy AC, Ashok A. Isolated tubercular osteomyelitis of scapula - A report of two cases and review of literature. J Orthop Case Rep 2013;3:7-11.

[8] Teo HE, Peh WC. Skeletal tuberculosis in children. Pediatr Radiol 2004;34:853-60.

[9] Martini M, Adjrad A, Boudjemaa A. Tuberculous osteomyelitis. A review of 125 cases. Int Orthop 1986;10:201-7.

[10] Khan SA, Zahid M, Sharma B, Hasan AS. Tuberculosis of frontal bone: a case report. Indian J Tub 2001;48:95-6.

[11] Tripathy SK, Sen RK, Sharma A, Tamuk T. Isolated cystic tuberculosis of scapula: case report and review of literature. J Orthop Surg Res 2010;5:72.

[12] Kam WL, Leung YF, Chung OM, Wai YL. Tuberculous osteomyelitis of the scapula. Int Orthop 2000;24:301-2. 